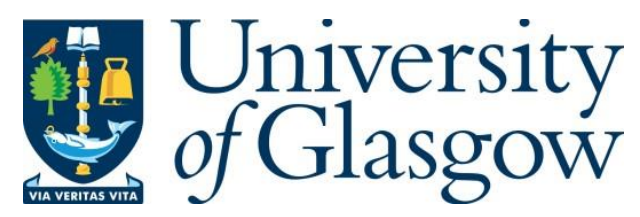

Ozioko, O. O., Navaraj, W. T., Yogeswaran, N., Hersh, M. and Dahiya, R. (2018)

Tactile Communication System for the Interaction between Deafblind and Robots. In:

27th IEEE International Symposium on Robot and Human Interactive Communication (RO-MAN), Nanjing, China, 27-31 Aug 2018, pp. 416-421. ISBN 9781538679814.

There may be differences between this version and the published version. You are advised to consult the publisher's version if you wish to cite from it.

http://eprints.gla.ac.uk/201386/

Deposited on: 18 November 2019

Enlighten - Research publications by members of the University of Glasgow http://eprints.gla.ac.uk 


\title{
Tactile Communication System for the Interaction between Deafblind and Robots
}

\author{
Oliver Ozioko $^{v \psi}$, William T. Navaraj ${ }^{\psi}$, Nivasan Yogeswaran ${ }^{\psi}$, Marion Hersh $^{v}$, Ravinder Dahiya ${ }^{\psi}$ \\ "Bendable Electronics and Sensing Technologies (BEST) group, School of Engineering, University of \\ Glasgow, UK. "Biomedical Engineering Division, School of Engineering, University of Glasgow, UK \\ *Correspondence to Ravinder.Dahiya@glasgow.ac.uk
}

\begin{abstract}
This paper presents a touch-based system for communication by deafblind people with humans and robots. With presented system the deafblind people can send control signal to robots by writing block letters on an array of 8 × 8 diamond-shaped capacitive touch sensors. For better conformability with curved surfaces, the touch sensors arrays have been fabricated on flexible polyvinyl chloride (PVC) substrate. The characters written by deafblind on the tactile sensing array are recognized by Convolution Neural Network (CNN), which is trained with data from Extended Modified National Institute of Standards and Technology (EMNIST) database. A mobile app was also developed to efficiently recognize and display the written letters. Finally, a Baxter robot was simulated using Robot Operating System (ROS) and was remotely controlled by deafblind through English block letters written on the tactile sensing array. The result shows that the robot could successfully execute the 'pick or drop' task. The developed system demonstrates the efficacy of tactile based communication by deafblind people to independently interact with a robot and their environment.
\end{abstract}

\section{INTRODUCTION}

The human-to-machine interaction methods developed so far generally depend on the use of auditory and vision sensory modalities. However, this does not work for deafblind people due to impaired hearing and vision sensing modalities. This necessitates the development of alternative tools that allow them to interact with real-world [1]. In the absence of these two major sensory modalities, the deafblind people have to depend on the sense of touch to communicate and interact with the environment [2]. Therefore, tactile interface becomes a critical component of any alternative tool meant for deafblind people to interact with robots and the real world both locally or remotely [3]. With robots entering into our daily lives in number of ways, the interaction of deafblind people with robots and machines also gains importance. A range of robots for rehabilitation, social and as household help is being explored currently to help the disabled and elderly [4]. The societal dependence on these machines for myriad tasks also necessitates the development of suitable tools for safe communication by the deafblind people. Indeed, the design and development of tools for deafblind people present a worstcase scenario due to significant reliance on one sensory modality i.e. tactile sensing. The work in this paper presents the development of a touch-based communication system for deafblind people. Although focus is on deafblind people, sighted and hearing people can adopt the scheme for interaction with robots also.
The manner in which deafblind people communicate using English block letters is shown in Fig. 1. The method involves deafblind person writing the English block letters with their finger on to the palm of the receiving person [5]. Here, the haptic feedback substitutes the impaired vision and auditory sensory modalities. These interactions may be accomplished by human, machine or both, and the environments can be real or virtual. The latter has recently changed the way humans interact with information and communicate ideas [6, 7]. Learning any new mode of communication or control strategy can be difficult and may require development of new training tools [8], and more so at old age. Considering this, the development of the interactive solution should be based on the mode of communication with which deafblind are familiar. Since, deaf blindness is mainly caused by old age, illness or accident (it is not always by birth) $[9,10]$, it is expected that deafblind people have prior knowledge of the English block letters. For this reason, as well as the fact the deafblind people already communicate using English block letters, the touchbased system presented here also uses the English block letters to communicate.

This paper reports for the first time a flexible touch-based communications system for robot-deafblind interaction using an array of capacitive touch-sensors in combination with Convolution Neural Network $(\mathrm{CNN})$ for recognition of the English block letters. The developed wearable tactile communication system comprises of an $8 \times 8$ diamond-shaped array of capacitive touch sensors. The touch sensor array is fabricated on flexible polyvinyl chloride (PVC) to allow them to conform to curved surfaces. The developed systems uses touch-based array to generate the English block letters, which

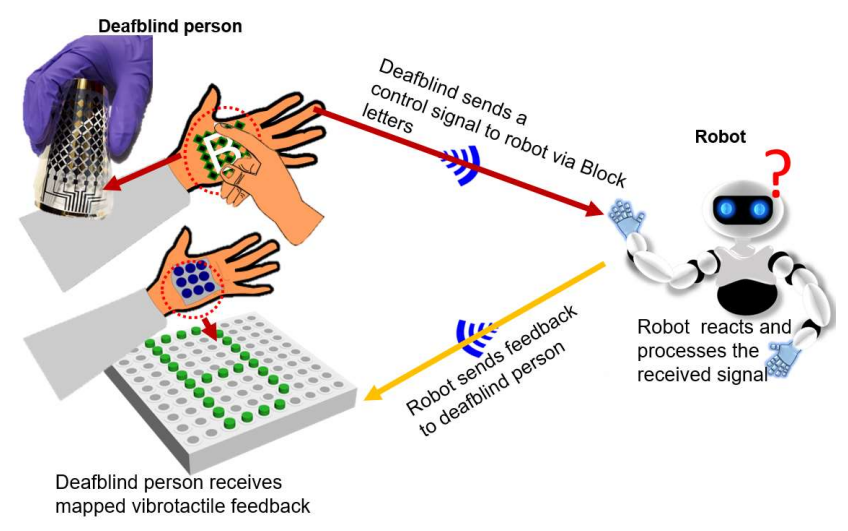

Figure 1. Schematic illustration of the tactile communication system for interaction of deafblind with robot. 
in turn are used to control the robots (Fig. 1). A CNN is also developed to facilitate the character recognition of the written block letters so that the robot can respond as per the character. Moreover, sending a character involves less data and therefore more efficient compared to sending data of each taxel elements.

This paper is organised as follows: The state of the art related to devices used for communication by the deafblind people is given in Section II. The description of the overall system is given in Section III. This is followed by the fabrication of the tactile sensing array in Section IV and the results related to testing of presented communication system including the training and implementation of $\mathrm{CNN}$ and the interface with a simulated Baxter robot are given in Section V. Finally, conclusion and the future outlook are given in Section VI.

\section{DeAfBlind COMmunicAtion - State of THE ART}

Depending on the extent and history of impairment as well as the country or region, a number of tactile and non-tactile communication methods are used by deafblind people [1]. The tactile methods include using block letters, deafblind manual alphabets (e.g. American and British deafblind manual alphabet, "Lorm, and Malossi alphabet), Braille, and so on. The non-tactile methods include speech, and sign language etc. While some of the deafblind people are born with this condition, a majority of them acquire deaf blindness at later stage in their life and therefore they are likely to have learnt some language (English, for instance) [1]. A number of deafblind people in this category generally use the English block letters to communicate [5]. The English block letter is a tactile deafblind communication method in which the deafblind person brings out the palm and the sender writes letters on it to communicate (Fig. 1) [9]. To reduce interdependence among deafblind people and to allow them to communicate independently, tactile sensing technologies are often employed in various assistive devices they use.

Tactile sensing involves the use of sensors to acquire tactile information through physical touch followed by measurement of properties such as temperature, vibration, pressure, texture, shape, and normal forces $[4,18,19]$. The sensing not only involves the detection and/or measurement of the spatial distribution of forces perpendicular to an area but also on the interpretation of the corresponding information [4,20-22]. Various types of tactile sensors reported previously involve several transduction methods such as capacitive [23], piezo-resistive [24], piezo-electric [25-27], optical [28] etc. In the case of deafblind people, several categories of tactile communication devices, with different tactile interfaces, have been developed and they are based on different deafblind communication methods. Table I presents a summary of existing devices for deafblind people and the tactile sensors used. It shows that early communication devices were mainly based on the American deafblind manual alphabet. They are hand-shaped devices with moveable fingers whose movement is controlled by a computer [2]. The listener touches the device to read the finger position. It is more of a mechanical device as it has no touch sensor embedded on the device. Handtapper was the first device to support British deafblind manual alphabet but was one-way and not wearable or portable.
TABLE 1: SUMMARY OF EXISTING TACTILE COMMUNICATION DEVICES FOR DEAFBLIND PEOPLE

\begin{tabular}{|c|c|c|c|c|}
\hline Comm. Strategy & Actuator used & Tactile Sensor & Year & Ref \\
\hline ADMA & None & None & 1987 & [11] \\
\hline ADMA $^{*}$ & None & None & 1994 & [12] \\
\hline Malossi Alphabet & Vibration motor & Tactile buttons & 2008 & [13] \\
\hline Lorm Alphabet & Vibration motor & $\begin{array}{l}\text { Capacitive -based } \\
\text { fabric Pressure sensors }\end{array}$ & 2012 & [14] \\
\hline $\mathrm{BDMA}^{+}$ & Vibration Motor & None & 2015 & [15] \\
\hline Braille & Vibration motor & Capacitive sensors & 2015 & [16] \\
\hline FingerBraille & Vibration Motor & Force Sensing Resistor & 2017 & {$[17]$} \\
\hline
\end{tabular}

Recently, portable two-way communication prototype devices with touch-sensors and other deafblind communication methods have been developed. Lorm glove was developed to support the Lorm alphabet commonly used in Austria, parts of Germany and Poland [29] - it uses a fabric pressure sensor as the touch sensor and vibration motor as a means of feedback to deafblind people. Another Lorm-based device called Lormer, involves users resting a hand on the dome of a special sieve and the letters communicated to them through a computer controlled jet of air from under the sieve [30]. DB-HAND for Malossi alphabet (used in Italy) is another alternative that uses tactile buttons as a touch sensor and also vibration motors as means of feedback to deafblind people [6]. A two-way communication device based on Finger Braille has also been reported [15]. None of these solutions employed the English block letters as a communication strategy for deafblind people nor means of interaction with robots.

Table I also shows that a number of the existing devices are based on capacitive sensors. In contrast to these existing deafblind communication devices, which use single touch sensor, the work presented here uses an array of capacitive touch sensors.

\section{DESCRIPTION OF THE OVERALl SyStem}

The block diagram of the overall approach is shown in Fig. 2. The developed system is targeted at a category of deafblind people who have acquired English language skills before they became deaf-blind. Overall, the deafblind user will be equipped with a wearable tactile sensing array for sending messages and tactile actuators for receiving messages/feedback. To send a message to a robot, the deafblind person writes the English block letters on the tactile array and receives feedback via vibrotactile feedback

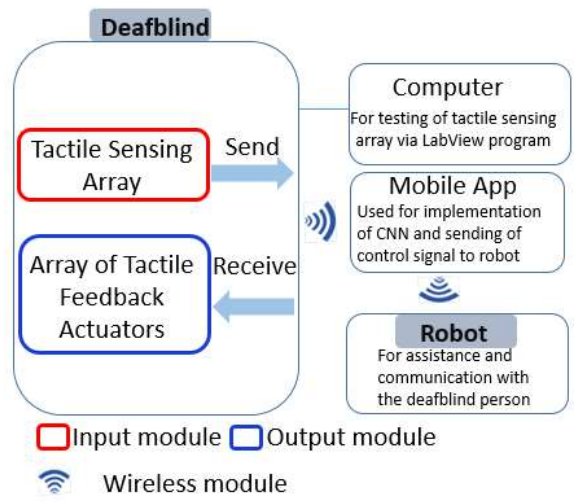

Figure 2. Block diagram of the overall tactile communication system 
actuators. The latter has not been implemented in this work. The system comprises of three main modules: (a) the input module, (b) the wireless module and (c) the output module. The input module is a capacitive touch-sensing array used for sending control signals to the robot. The output module is an array of vibrotactile feedback actuators each equipped with a movable skin contactor. This module is used for receiving messages from the robot. A Bluetooth module was used for wireless communication. When a deafblind person draws a particular block letter on the touch-sensors array, it is coded and sent wirelessly as control command to a nearby robot via a mobile app that uses CNN. When the robot sends feedback to the deafblind user, the actuator vibrates, and the contactors moves in correspondence to the English block letter. So, the deafblind person is able to feel the array of contactors to decode the message. The computer block in Fig. 2 represents the setup to test the output of the capacitive tactile array readout circuit. This work discusses only the implementation of the array of capacitive touch-sensors and the accompanying $\mathrm{CNN}$ for character recognition. Work on the development of the array of tactile feedback actuators is currently on-going.

\section{TACTILE SENSING ARRAY}

\section{A. Capacitive Touch Sensor Array}

The touch-sensors array is based on the capacitive sensing similar to the implementation reported in [23]. The response of the touch sensors was converted from capacitive variation to a voltage through a circuit interface shown in Fig. 3 and this is based on (1).

$$
C=I_{C}(\Delta V / \Delta t)^{-1}
$$

Where, $C$ is the capacitance of touch sensor, $I_{C}$ is the constant current through the capacitor, $\Delta V$ is the change in voltage, and $\Delta t$ is the change in time. The readout circuit uses a $18 \mathrm{~F} 45 \mathrm{~K} 22$ PIC microcontroller to process the row and column capacitance values to determine location of touch or contact on the array. For testing, these values were read into a LabVIEW program running in a computer via the serial port using MAX 232. For communication, the microcontroller sends data wirelessly through a Bluetooth module to the mobile phone, which implements the $\mathrm{CNN}$ and triggers the controlling of the robot.

\section{B. Fabrication of Touch Sensor Array}

The touch sensor array was developed on a flexible PVC substrate. The fabrication process flow is schematically shown in Fig. 4(a)-(f) and the fabricated tactile sensing array is shown in Fig. 4g. The sensor has column electrodes deposited on one side of the PVC and row electrodes on the reverse side. The deposition of the row and column electrodes were carried out using hard masks developed in-house. Plassys MEB 550S Electron Beam Evaporator system was used to deposit the electrodes $(20 / 120 \mathrm{~nm} \mathrm{NiCr} / \mathrm{Au})$ on both side of the substrate thereby resulting in the row and column electrode configuration. After the deposition of the electrodes, the hard mask was removed, and the surface of the sensor laminated with another piece of PVC sheet to protect the electrodes.

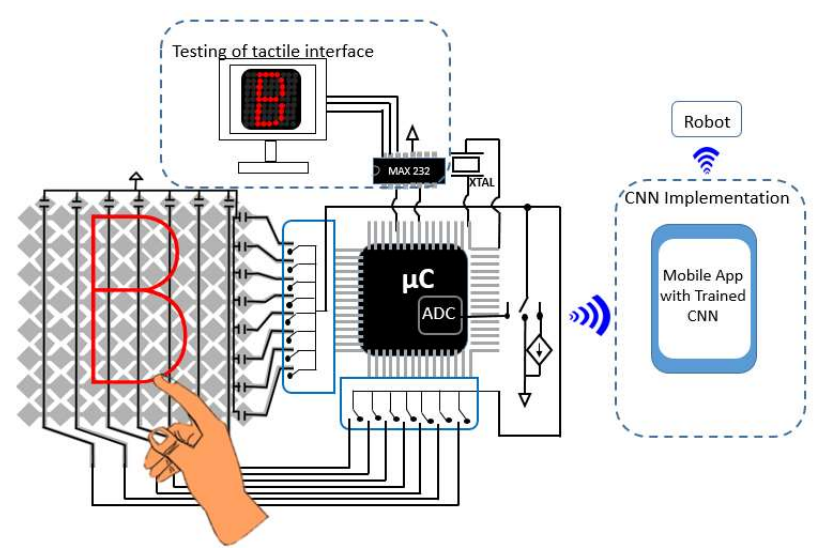

Figure 3. Readout schematics for the capacitive tactile sensing array
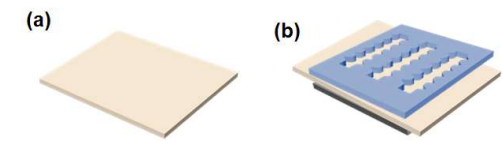

$\mathrm{NiCr}$ Seed layer $+\mathrm{Au}$ (c)

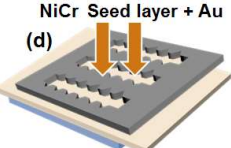

(e)
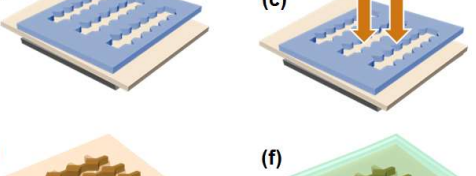

$\square$ Substrate $\square$ Mask (Column) $\square$ Mask (Row) $\square$ Deposited metal $\square$ Laminating sheet

$(\mathrm{g})$

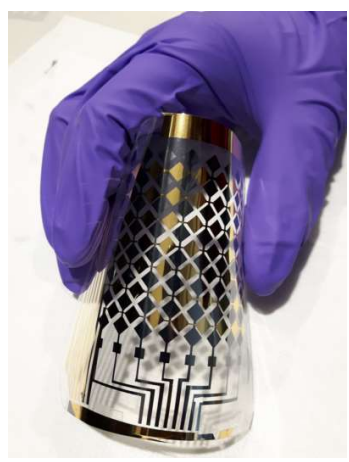

Figure 4. The fabrication steps and the fabricated touch sensor array. (a) Initial flexible PVC substrate (b) Alignment of shadow-mask on the top and bottom of the PVC substrate (c) Deposition of column electrodes (20/120nm $\mathrm{NiCr} / \mathrm{Au}$ ) (d) Deposition of row electrodes (20/120nm NiCr/Au) (e) Removal of shadow mask (f) PVC lamination (g) Fabricated capacitive sensor array.

\section{DeVice Testing And Results}

The performance of the implemented system was evaluated using four main tests: (1) Evaluation of the response of each pixel on the sensor array; (2) Evaluation of the feasibility of the system for Block letter communication; (3) Evaluation of the performance of $\mathrm{CNN}$; (4) Control of a simulated Baxter robot using the English block letters.

The tactile sensor array was interfaced to a LabVIEW program through a custom-developed hardware as shown in Fig. 5. The program's user interface has two main windows: (a) an array of points that corresponds to locations on the ensor array, and (b) A decay graph. Both windows were used to show the sensor's response to a touch event. 


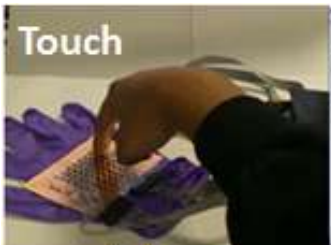

(a)

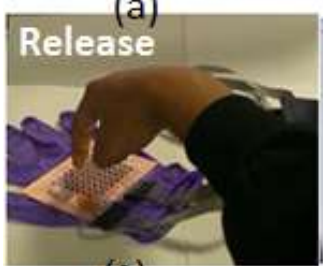

(c)

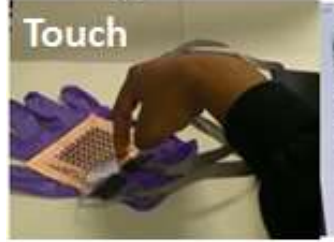

(e)

Figure 5. Testing of the response of various taxels of the sensor array

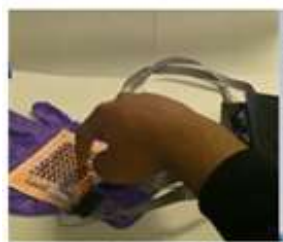

(a)

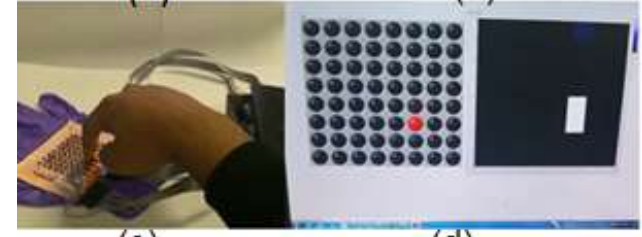

(c)

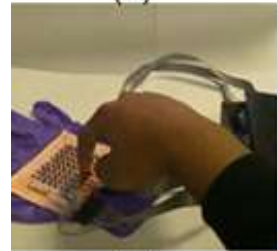

(e)

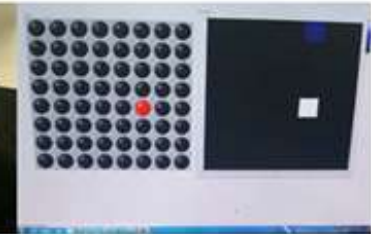

(b)

(d)

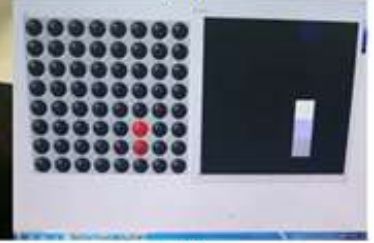

(f)

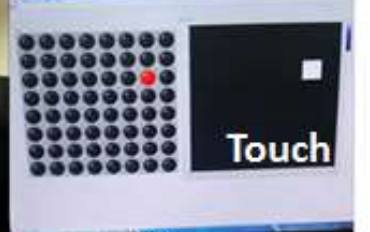

(b)

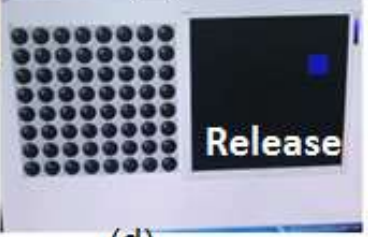

(d)

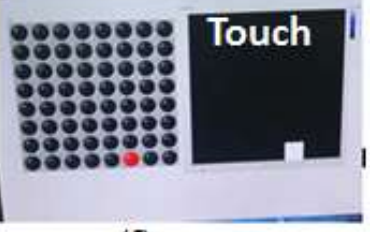

(f)
Figure 6. Evaluation of the system for writing and communicating with the English letter 'i'

A touch event is represented in the LabVIEW program by: (i) A red dot in the in-pixel map; (ii) a white square in a decay graph which gradually turns blue upon the release event. The performance of the implemented system upon touch and release event at various taxels is shown in Fig. 5(a)-5(f). Further, testing was carried out to analyse feasibility of the system for tactile communication. This was done by writing English alphabets on the touch sensor array. As a representation, the result of writing letter " $i$ " on the array is depicted in Fig. 6.

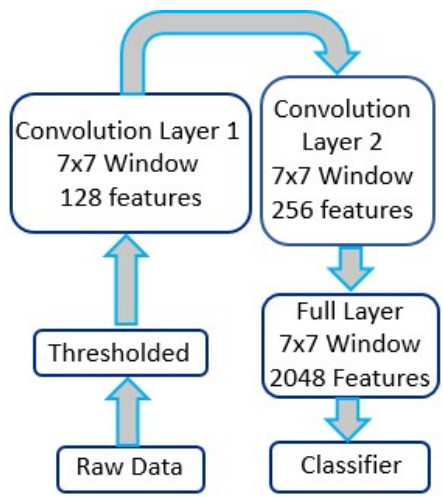

Figure 7. Block diagram showing different layers of the implemented Convolution neural network

\section{A. Convolution Neural Network - Training and Implementation}

To efficiently recognise the written English block letters, a CNN was trained using the EMNIST database to recognize the English block letters drawn on the tactile array. EMNIST is the extended version of Modified National Institute of Standards and Technology (MNIST) and was first introduced by Cohen et al. [31] for recognition of handwritten characters. EMNIST was used because it's a database comprising both numbers and letters, unlike MNIST which has only numbers. In [32], we have used Simbrain for simulating a Nano Wire Field Effect Transistor (NWFET) - based neural element with Multi-Layer Perceptron (MLP) for tactile data processing. Since MLPs are not efficient for character recognition and SimBrain cannot handle larger number of neurons, we have used TensorFlow to implement $\mathrm{CNN}$ to process data in a mobile phone. The training was carried out using a desktop computer and the resulting $\mathrm{NN}$ works on a mobile app for character recognition.

The layers of $\mathrm{CNN}$ are shown in Fig. 7. An 8 x 8 array was implemented to conform to the size of the touch-sensor array. EMNIST dataset comprise of $28 \times 28$ pixels dataset. To be able to classify data efficiently, the $8 \times 8$ taxel array output was thresholded, after the decay filter and up-sampled to $28 \times 28$ pixels. The up-sampled data was then fed into the network as raw data. The data is split into $7 \times 7$ window which is passed through the convolution layer $1(128 \mathrm{o} / \mathrm{p}$ features), convolution layer 2(256) features, fully connected layer (2048 features), with the number of features in a particular layer serving as the input for the next layer. Finally, the data was classified according to the corresponding character using the EMNIST database. The mobile app displays the recognized block letter when a closely related pattern is drawn on the array and sends it to the robot.

In addition to evaluation of the touch sensors array, the performance of CNN was equally evaluated, and the result of the training and implementation are shown in Fig. 8. 10,000 samples were used for training and 3,000 samples for the testing. The training accuracy reached $100 \%$ while the average test accuracy was $91 \%$. A mobile app was created and used for the implementation of CNN. When a pattern is drawn on the tactile array, the app uses $\mathrm{CNN}$ to decode and display the letter corresponding to the drawn pattern. As an example, each letter of the word "BEST" were written one at a time and the result is shown in Fig. 8. 


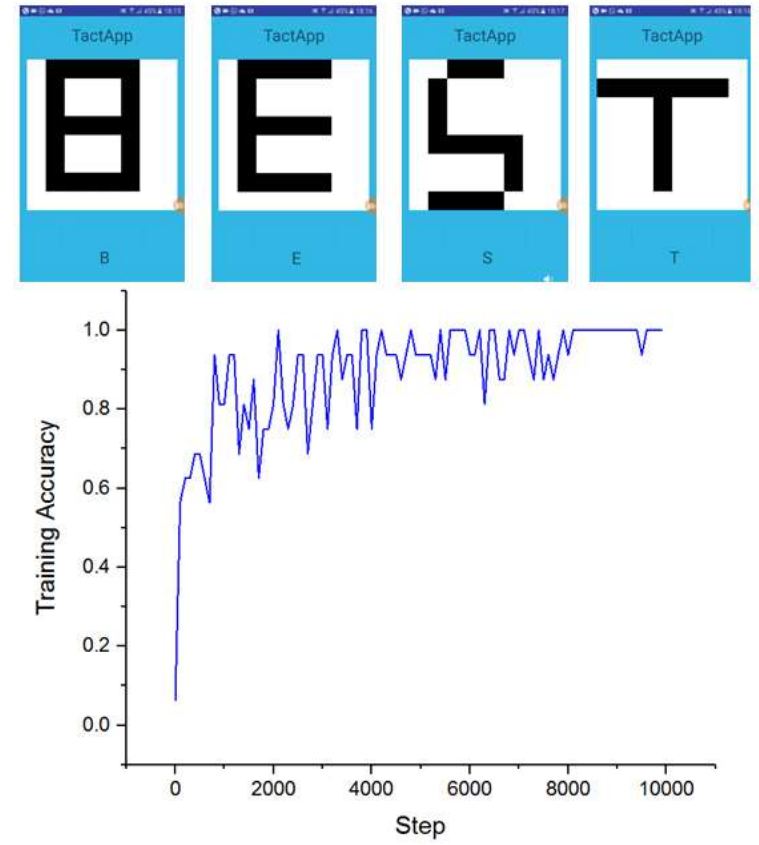

Figure 8. Training and character recognition of the letters of the word "BEST" by the mobile app given as input to the tactile sensor array.

\section{B. Interface with simulated robot environment}

A Baxter robot [33] was simulated using the Robot Operating System to pick and drop an object. Using the developed communication system, it was then controlled remotely using the developed tactile communication system to pick and drop an object as shown in Fig. 9. Letter "P" and " $D$ " was programmed to make the robot pick and drop an object respectively. So, with this, the Baxter robot was made to successfully carry out a "pick-and-drop" action using the developed communication system.

\section{CONCLUSION AND FUTURE WORK}

A wearable tactile communication system for robotdeafblind interaction is presented. Convolution neural network was trained and implemented to recognize the English block letters written on the array of tactile sensors. The performance of the system was demonstrated by controlling a Baxter robot using the English block letters. This shows that the system worked and will enable deafblind people to interact with robot and their environment by writing English block letters on a tactile sensor array and receiving feedback using array of tactile feedback actuators. Only the tactile sensing array was implemented in this work and future work will involve implementing the array of tactile feedback actuators to help the deafblind to receive response from robots, followed by end-user testing.

\section{ACKNOWLEDGMENT}

This work was supported in part by Engineering and Physical Sciences Research Council (EPSRC) Fellowship for Growth - Printable Tactile Skin (EP/M002527/1), and Tertiary Education and Trust Fund (TETFund), Nigeria. We are also thankful to Electronic Systems Design Centre (ESDC) for the support in terms of device design and characterization.

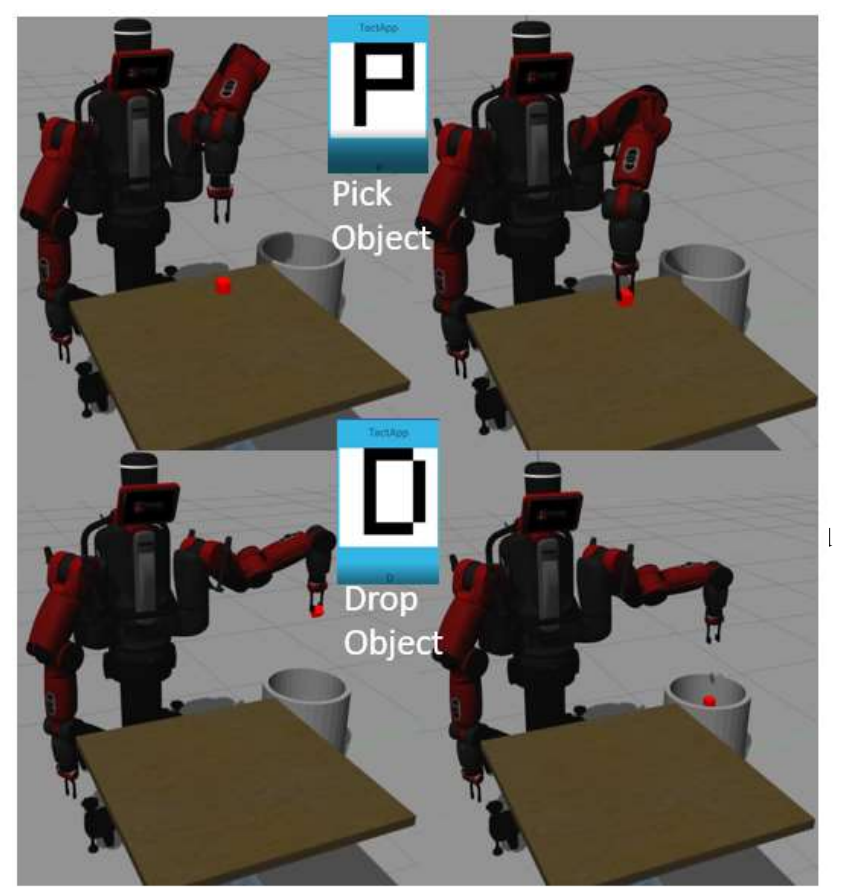

Figure 9. Controlling a simulated Baxter robot for a "pick-and-drop" action using the developed tactile communication system. $\mathrm{P}=$ Pick, $\mathrm{D}=$ Drop.

\section{REFERENCE}

[1] F. Sorgini, R. Caliò, M. C. Carrozza, C. M. Oddo, "Haptic-assistive technologies for audition and vision sensory disabilities," Disability and Rehabilitation: Assistive Technology, vol. 13, pp. 394-421, 2018.

[2] M. A. Hersh, M. A. Johnson, Eds., Assistive Technology for the Hearing-impaired, Deaf and Deafblind. Springer-Verlag London, 2003.

[3] R. Dahiya, W. Taube, S. Khan, E. Polat, "Developing electronic skin with the sense of touch," Information Display, vol. 31, pp 6-10, 2015.

[4] R. S. Dahiya, M. Valle, Robotic Tactile Sensing: Technologies and System: Springer Netherlands, 2012.

[5] J. Dammeyer, F. Ask Larsen, "Communication and language profiles of children with congenital deafblindness," British J. of Visual Impairment, vol. 34, pp. 214-224, 2016.

[6] M. A. Srinivasan, C. Basdogan, "Haptics in virtual environments: Taxonomy, research status, and challenges," Computers \& Graphics, vol. 21, pp. 393-404, 1997.

[7] M. Sreelakshmi, T. D. Subash, "Haptic Technology: A comprehensive review on its applications and future prospects," Materials Today: Proceedings, vol. 4, pp. 4182-4187, 2017.

[8] A. Vilouras, H. Heidari, W. T. Navaraj, R. Dahiya, "At-Home Computer-Aided Myoelectric Training System for Wrist Prosthesis," in Proceedings of Eurohaptics, 284-293, 2016.

[9] J. Dammeyer, "Deafblindness: A review of the literature," Scandinavian J. of Public Health, vol. 42, pp. 554-562, 2014.

[10] J. M. Schneider, B. Gopinath, C. M. McMahon, S. R. Leeder, P. Mitchell, J. J. Wang, "Dual Sensory Impairment in Older Age," J. of Aging and Health, vol. 23, pp. 1309-1324, 2011.

[11] M. Alexander, "Dexter - a finger-spelling hand for the deaf-blind," IEEE Robotics and Automation. Proceedings, pp. 1192-1195, 1987.

[12] D. L. Jaffe, "Evolution of mechanical fingerspelling hands for people who are deaf-blind," J. Rehabilitation Research and Development, vol. 31, pp. 236-244, 1994.

[13] N. Caporusso, "A Wearable Malossi Alphabet Interface for Deafblind People," in AVI '08 Proceedings of the working conference on Advanced visual interfaces, Napoli Italy, pp. 445-448, 2008.

[14] G. Ulrike, B. Tom, J. Gesche, "Mobile Lorm Glove - Introducing a Communication Device for Deaf-Blind People," TEI, pp. 127 - 130, 2012. 
[15] O. Ozioko, M. Hersh, "Development of a portable two-way communication and information device for deafblind people," In 13th AAATE International Conference, 2015.

[16] T. Choudhary, S. Kulkarni, P. Reddy, "A Braille-based mobile communication and translation glove for deaf-blind people," in Int. Conf. on Pervasive Computing (ICPC), pp. 1-4, 2015.

[17] O. Ozioko, W. Taube, M. Hersh, R. Dahiya, "SmartFingerBraille: A tactile sensing and actuation based communication glove for deafblind people," in 26th IEEE Int. Symp. on Ind. Electr., pp. 2014-2018, 2017.

[18] M. I. Tiwana, S. J. Redmond, N. H. Lovell, "A review of tactile sensing technologies with applications in biomedical engineering," Sensors and Actuators A: Physical, vol. 179, pp. 17-31, 2012.

[19] Y. Wan, Y. Wang, C. F. Guo, "Recent progresses on flexible tactile sensors," Materials Today Physics, vol. 1, pp. 61-73, 2017.

[20] P. S. Girão, P. M. P. Ramos, O. Postolache, and J. M. D. Pereira, "Tactile sensors for robotic applications," Measurement, vol. 46, pp. 1257-1271, 2013.

[21] S. Luo, J. Bimbo, R. Dahiya, H. Liu, "Robotic Tactile Perception of Object Properties: A Review," Mechatronics 48, 54-67, 2017.

[22] R. S. Dahiya, G. Metta, M. Valle, G. Sandini, "Tactile sensing-from humans to humanoids," IEEE Trans. Robotics, vol. 26, pp. 1-20, 2010.

[23] C. G. Núñez, W. T. Navaraj, E. O. Polat, R. Dahiya, "EnergyAutonomous, Flexible, and Transparent Tactile Skin," Adv. Funct Mater., vol. 27, 2017

[24] M. Ahmed, M. M. Chitteboyina, D. P. Butler, Z. Çelik-Butler, "MEMS force sensor in a flexible substrate using nichrome piezoresistors," IEEE Sensors J., vol. 13, pp. 4081-4089, 2013.

[25] R. Dahiya, A. Adami, L. Pinna, C. Collini, M. Valle, L. Lorenzelli, "Tactile Sensing Chip with POSFET Arrays and Integrated Interface Electronics," IEEE Sensors J., vol. 14, pp. 3448-3457, 2014.

[26] S. Gupta, L. Lorenzelli, R. Dahiya, "Multifunctional flexible PVDFTrFE/BaTiO based tactile sensor for touch and temperature monitoring," in IEEE Sensors, pp. 1-3, 2017.

[27] S. Hannah, A. Davidson, I. Glesk, D. Uttamchandani, R. Dahiya, H. Gleskova "Multifuncational sensor based on organic field effect transistor and ferroelectric poly(vinylidene fluoride trifluoroethylene," Organic Electronics, vol. 57, 170-177, 2017.

[28] J.-S. Heo, J.-H. Chung, J.-J. Lee, "Tactile sensor arrays using fiber Bragg grating sensors," Sensors and Actuators A: Physical, vol. 126, pp. 312-327, 2006.

[29] O. Satoshi, H. Sadao, S. Nobuyuki, H. Tetsumi, "The Introduction of Tele-Support System for Deaf-blind People using Body-Braille and a Mobile Phone," in 5th IEEE Consumer Communication and Networking Conf., Las Vegas, Nevada, pp. 1263 - 1264, 2008.

[30] R.-G. Fernando, M. O. Cesar, F. Alejandro, C. H. Joel, "My Vox Device for the Communication between People: Blind, Deaf, Deafblind, and Unimpaired," IEEE pp. 506-510, 2014.

[31] G. Cohen, S. Afshar, J. Tapson, A. V. Schaik, "EMNIST: Extending MNIST to handwritten letters," in Int. Joint Conf. on Neural Networks (IJCNN), pp. 2921-2926, 2017.

[32] W. T. Navaraj, C. G. Núñez, D. Shakthivel, V. Vinciguerra, F. Labeau, D. H. Gregory, et al., "Nanowire FET Based Neural Element for Robotic Tactile Sensing Skin," Frontiers in Neuroscience, vol. 11, 501, 2017. 